\title{
Symptom Management for Patients with Advanced Gastrointestinal Cancer Undergoing Chemotherapy Based on Patient-Reported Outcomes Obtained from Electronic Platforms
}

\section{Xuyuan Dong}

Xi'an Jiaotong University Medical College First Affiliated Hospital Department of Medical Oncology https://orcid.org/0000-0003-2037-7852

Huili Wu

Xi'an Jiaotong University Medical College First Affiliated Hospital Department of Medical Oncology Yangwei Fan

Xi'an Jiaotong University Medical College First Affiliated Hospital Department of Medical Oncology Miaomiao Zhang

Xi'an Jiaotong University Medical College First Affiliated Hospital Department of Medical Oncology

\section{Xiaofang Chen}

Xi'an Jiaotong University Medical College First Affiliated Hospital Department of Medical Oncology

Yinying Wu ( $\square$ shadowless_111@163.com)

Xi'an Jiaotong University Medical College First Affiliated Hospital Department of Medical Oncology https://orcid.org/0000-0003-3075-800X

\section{Research Article}

Keywords: electronic system, patient-reported outcomes, advanced gastrointestinal cancer, symptom management, QoL

Posted Date: September 29th, 2021

DOl: https://doi.org/10.21203/rs.3.rs-821631/v1

License: (c) (1) This work is licensed under a Creative Commons Attribution 4.0 International License. Read Full License 


\section{Abstract}

Purpose: The electronic patient-reported outcome (ePRO) system plays an important role in routine cancer treatment, but information on its use to assess and monitor symptoms and quality of life (QoL) in advanced gastrointestinal cancer patients in China is limited.

Methods: Sixty patients with advanced gastrointestinal cancer at the First Affiliated Hospital of Xi'an Jiaotong University were enrolled. They completed the ePRO questionnaires on a computer to provide nutrition, appetite, psychology, and QoL data at the beginning of cancer treatment. The nurses followed up the assessment results and provided feed-back to the clinicians, then, doctors addressed the symptoms according to the assessment results. The primary outcome was change in nutritional status. Secondary endpoints included appetite, psychological status and QoL.

Results: There were 60 patients, 54 completed the follow-up. At the fourth visit, the Nutrition Risk Screening 2002 (NRS2002) and Patient-Generated Subjective Global Assessment (PG-SGA) scale scores improved significantly $(p<0.05)$. More than 70\% of PG-SGA $(73.08 \%)$, Functional Assessment of Anorexia/Cachexia Therapy Anorexia/Cachexia Subscale (FAACT-A/CS) (76.92\%), Psychological Distress Thermometer (DT) (75.00\%) and EuroQol Five Dimensions Questionnaire (EQ-5D) (78.84\%) scores improved or remained stable. The results of the Generalized Linear Model (GLM) analysis showed that nutritional status, psychological status and QoL gradually improved with symptom management $(p<$ 0.05).

Conclusion: This symptom management closed-loop model can effectively manage the relevant symptoms of patients and improve QoL.

Clinical trial information ChiCTR2100048290, date of registration July 5, 2021.

\section{Background}

Gastrointestinal cancer is highly prevalent in China. According to the latest cancer burden data released by the International Agency for Research on Cancer (IARC), new cases of gastrointestinal tumors in China exceeded 1.3 million ${ }^{[1]}$ in 2020 . Systemic chemotherapy is the main treatment for patients with advanced cancer, and during the treatment period, patients are prone to problems such as malnutrition, anorexia, depression, and anxiety, which not only reduce QoL, but also affect the antitumor therapy results and survival prognosis ${ }^{[2-3]}$. Traditional symptom management mainly relies on clinicians, who do not actively monitor symptoms and often ignore patients' feedback ${ }^{[4]}$.

"Patient-centered" therapy has been advocated in the context of antitumor therapy in recent years, patientcentered therapy not only focuses on overall survival (OS), but also attaches increasing importance to QoL and patients' feelings and participation. As a new clinical outcome, patient-reported outcomes (PROs) are reports about a patient's health condition received directly from the patient, without interpretation by other parties, that reflect the subjective feelings of the patient ${ }^{[5]}$. PROs measure patients' 
daily function, symptoms, QoL and so on, through various validated scales, which are completed by the patients themselves. Compared with the judgment of medical staff, PRO measures are more accurate and sensitive, have attracted increasing clinical workers' attention and recognition and have been widely used in many clinical studies ${ }^{[4,6]}$. Cleeland et al ${ }^{[7]}$ conducted a randomized controlled study of PRO for symptom management in 100 patients undergoing thoracotomy. The patients were randomized to the PRO group or the traditional group. Finally, it was found that PRO-based symptom management could reduce the severity of symptoms within 4 weeks after discharge. Ethan et al ${ }^{[8]}$ conducted a symptommonitoring study via PROs in patients treated with chemotherapy. The results showed that PRO-based active monitoring can allow for the timely management of various symptoms, improve QoL and prolong patient survival by 5.2 months compared with traditional passive monitoring.

Symptom monitoring during chemotherapy is essential and is the cornerstone of medical oncology practice ${ }^{[9]}$, and PRO-based symptom management can not only better monitor symptoms, and improve QoL and satisfaction with treatment ${ }^{[10-11]}$ but also contribute to improvements in clinical outcomes ${ }^{[4]}$. Shen Lin et al ${ }^{[12]}$ conducted a PRO-based trial to explore the survival benefit of early interdisciplinary supportive care combined with first-line standard treatment for patients with advanced esophagogastric cancer (ESC). The results showed that after 9 weeks of multidisciplinary symptom management, nutritional and psychological status, emotional functioning and cognitive functioning in the ESC group were significantly improved, median OS was significantly prolonged, and the risk of death was significantly reduced by $32 \%$. The trial showed that early nutritional and psychological support therapy can provide significant survival benefits to patients with ESC.

By collecting health information directly from patients through PROs and combining it with other clinical indicators, the patients' condition and treatment effect can be more holistically and objectively evaluated. PROs emphasize the importance of patients' feelings and input, and is the clinical implementation of the "patient-centered" concept. However, at present, PROs are still not widely used in China, due to some of the following limitations: medical staff have insufficient knowledge and skills related to PROs and do not fully realize their value; patients do not pay enough attention to their own symptoms and have poor compliance in completing paper scales; and the production, completion, data entry and statistical analyses associated with the paper form required for PRO assessment are laborious. Therefore, it is necessary to provide corresponding education to promote attention to PROs and develop convenient and comprehensive digital tools to promote the clinical application of PROs. With the rapid development of digital technology and the popularization of network information equipment, ePROs, which use internet or telephone methods for self-reporting, have emerged ${ }^{[13]}$. With timely management and regular monitoring of the patient's relevant symptoms, functions and needs, ePROs can better improve the efficiency and quality of symptom management and further improve patient satisfaction. Therefore, we plan to cooperate with the Department of Epidemiology and Statistics, School of Public Health, Xi'an Jiaotong University to construct an ePRO system using the REDCap platform to realize the functions of electronic information collection, evaluation, data storage and real-time access. 
Based on the fact that patients with advanced gastrointestinal cancer have a heavy burden of symptoms and the current clinical symptom management relies on doctors, we plan to use ePROs to evaluate and monitor patients' nutritional status, appetite, and psychology, aiming to establish an effective management model of related symptoms, to improve patient QoL and increase clinical benefits.

\section{Patients And Methods}

\subsection{Study Design and Participants}

This study was a single-center study performed at the Department of Medical Oncology of the First Affiliated Hospital of Xi'an Jiaotong University.

Only inpatients were considered for inclusion. Eligible patients had histologically or cytologically confirmed gastrointestinal cancer, had a baseline Karnofsky Performance Status (KPS) $>70$ points, had an estimated life expectancy of more than 6 months, had adequate cognitive and reading abilities and had good compliance.

This study was approved by the Ethics Committee of the First Affiliated Hospital of Xi'an Jiaotong University. All observed subjects were fully informed and volunteered to participate.

\subsection{Methods}

\subsubsection{PRO Scales}

The primary outcome was nutritional status. Secondary endpoints included appetite, psychology and QoL. These indicators were assessed via widely validated scales. (1) Nutritional status was assessed as follows: Nutritional risk was screened with NRS $2002^{[14]}$, which includes four items: primary screening, disease status, nutritional status and age. Assessment results are divided into two categories: no nutritional risk ( $₫ 3$ points) and nutritional risk ( $\geq 3$ points). Nutritional assessment was carried out with PG-SGA ${ }^{[15]}$, which includes seven items: weight, food intake, symptoms, activities and function, disease and its relation to nutritional requirements, metabolic demand, and physical characteristics, based on the total score, individuals are divided into three categories: normal nutrition (0-3 points), moderate malnutrition (4-8 points) and severe malnutrition (> 8 points). (2) Appetite status was assessed as follows: appetite assessment was performed using FAACT-A/CS ${ }^{[16]}$, and dietary status was assessed with the Brief Dietary Self-Assessment Scale (northwest version) ${ }^{[17]}$. The FAACT-A/CS consists of 12 items, with a total score of 48 points. Verified by the University of Amsterdam Medical Center in the Netherlands, a cutoff value of $\leq 37$ has been established for anorexia [18]; the Brief Dietary SelfAssessment Scale ${ }^{[17]}$ is a dietary self-assessment tool developed by Professor Cong Minghua's team, and patients can conduct self-evaluation based on the tool and their dietary characteristics, with scores ranging from 1-5. (3) Psychological screening was conducted as follows: DT was used for rapid identification of psychological distress ${ }^{[19]}$, which is recommended by the National Comprehensive 
Cancer Network (NCCN) guidelines and consists of two parts: a single item scale from 0 to 10 (0 represents no pain; 10 represents extreme pain) and a list of questions containing 34 items. (4) QoL was assessed as follows: EQ-5D ${ }^{[20-21]}$ was used for assessment, which is composed of two parts: the descriptive system and EQ-VAS. The descriptive system assesses five items (mobility, self-care ability, daily activities, pain/discomfort and anxiety/depression), which are converted into index scores, with a range of -1 to 1 , where 0 represents death, 1 represents complete health, and negative values mean health status worse than death; the EQ-VAS is between 0 and 100, representing the general health status of the population.

\subsubsection{Construction of ePRO System}

The ePRO system was designed and constructed by the Department of Epidemiology and Statistics, School of Public Health, Xi'an Jiaotong University according to standard procedures using the REDCap (Research Electronic Data Capture) platform, with multiple functions, such as scale filling, real-time statistical scores, data storage and export, and user authority management. The electronic scales are all multiple-choice. The system is standardized, reusable, safe, convenient and easy to understand and access. The REDCap platform ${ }^{[22]}$ was developed by the team of Professor Paul Harris of Vanderbilt University in 2004. It is a mature and secure online clinical research and trial database network application that collects and manages almost all types of research data and information. Since 2006, it has been freely available to clinical research institutions in the United States and other countries.

\subsubsection{PRO Assessment and Intervention}

After enrollment, the nurses trained the patients to log in and use the ePRO system with a project-specific tablet computer. The assessment was performed on the first day of each chemotherapy cycle. The patients completed electronic scales to assess nutritional status, appetite, psychology, QoL and other conditions. Patients were intended to complete no less than 4 assessments.

After the completion of the self-reported scales, the nurses conducted follow-up on the scale scores. For those with abnormal scores, the nurses would provided feed-back to the doctors for further diagnosis and intervention. Multidisciplinary nutritional interventions, anorexia interventions and psychotherapy were initiated according to the assessment results. In addition, every month, the department would carry out 1-2 patient education activities to share content such as reasonable home diet, and nutritional supplements through online and offline methods.

\subsubsection{Statistical analysis}

Statistical analysis was performed using the statistical software SPSS 22.0. Measurement data are expressed as the mean \pm standard deviation (mean $\pm \mathrm{sd}$ ). Comparisons were performed by paired $t$ test. The GLM was used to analyze the change trend of various indicators affected by the number of visits. Two-sided $p$ values of less than 0.05 were considered to indicate statistical significance.

\section{Results}




\subsection{Baseline Characteristics}

A total of 60 patients were enrolled from July 2020 to August 2020, and the average age of these patients was $55.72 \pm 10.21$ years. The baseline data are shown in Table 1 .

Table 1

Baseline characteristics

\begin{tabular}{|lll|}
\hline Feature & N & Percent (\%) \\
\hline Sex & & \\
\hline Male & 41 & 68.3 \\
\hline Female & 19 & 31.7 \\
\hline Cancer metastasis & & \\
\hline Yes & 35 & 58.3 \\
\hline No & 25 & 41.7 \\
\hline Cancer type & & \\
\hline Gastric cancer & 21 & 35.0 \\
\hline Esophageal cancer & 3 & 5.0 \\
\hline Pancreatic cancer & 8 & 13.3 \\
\hline Colorectal cancer & 23 & 38.3 \\
\hline Liver cancer & 2 & 3.3 \\
\hline Gallbladder cancer & 1 & 1.7 \\
\hline Other & 2 & 3.3 \\
\hline Cancer stage & & \\
\hline$\square$ & 2 & 3.3 \\
\hline$\square$ & 9 & 15.0 \\
\hline$\square$ & 19 & 31.7 \\
\hline$\square$ & 30 & 50.0 \\
\hline
\end{tabular}

\subsection{Results of the First Visit}

At baseline, 60 patients had completed data for body weight, body mass index (BMI), KPS and PRO scales (Table 2). The average NRS2002 score was $2.97 \pm 0.93$, and $61 \%$ of patients had a score $\geq 3$ 
points, indicating that more than half of the patients were at nutritional risk. The average score of the PGSGA was $7.73 \pm 4.70$, suggesting that the patients' overall nutritional status was poor. The mean scores of FAACT-A/CS and the Brief Dietary Self-Assessment Scale were $34.64 \pm 7.48$ and $3.86 \pm 0.90$, respectively, showing that the patients' appetite and food intake were not optimal. These results indicate that patients with malignant tumors are prone to problems such as malnutrition and anorexia. Early diagnosis as well as treatment are needed.

Table 2

Results of first visit

\begin{tabular}{|ll|}
\hline Metrics & Mean \pm SD \\
\hline Weight & $59.80 \pm 8.16$ \\
\hline BMI & $21.28 \pm 2.86$ \\
\hline KPS & $89.17 \pm 2.79$ \\
\hline Nutritional status assessment & \\
\hline PG-SGA & $2.97 \pm 0.93$ \\
\hline Appetite assessment & $7.73 \pm 4.70$ \\
\hline FAACT-A/CS & \\
\hline Brief Dietary Self-Assessment Scale & $3.86 \pm 0.90$ \\
\hline Psychological assessment & \\
\hline DT & $2.25 \pm 1.86$ \\
\hline QoL assessment & \\
\hline EQ-5D index score & $0.92 \pm 1.33$ \\
\hline EQ-VAS & $80.75 \pm 15.25$ \\
\hline
\end{tabular}

\subsection{Changes in Results at the Fourth Visit}

A total of 60 patients were enrolled, 6 patients were lost to follow-up, and 54 patients completed the fourth visit assessment. NRS 2002 and PG-SGA showed significant improvements compared with their values at the first visit ( $p=0.017$ and 0.007 , respectively), suggesting that this symptom management model can significantly improve patients' nutritional status. The FAACT-A/CS score increased by 2.31 points $(p=0.014)$, indicating that the patients' appetite was significantly improved. The DT score decreased by 1.08 points $(p<0.001)$, indicating that the patients' psychological distress symptoms were significantly reduced. The index score and VAS were significantly improved $(p=0.037$ and 0.004 , 
respectively), suggesting that the QoL of patients can be remarkably improved after 3 cycles of symptom management. Other indicators showed a tendency to be maintained or improved. The results are detailed in Table 3.

Most patients' symptoms improved or were maintained at the fourth visit. More than $70 \%$ of PG-SGA (73.08\%), FAACT-A/CS (76.92\%), DT (75.00\%) and EQ-5D (78.84\%) scores were improved or unchanged. The proportion of patients whose body weight and BMI were improved or maintained was $63.46 \%$, while the number of patients whose diet was improved or maintained exceeded $55.77 \%$. The results are shown in Fig. 1.

Table 3

Paired t-test results of the fourth visit and the first visit assessment

\begin{tabular}{|llll|}
\hline Metrics & Diff. & t value & $\boldsymbol{p}$ value \\
\hline Weight & $-0.17(-1.12 \sim 0.78)$ & -0.36 & 0.720 \\
\hline BMI & $0.02(-0.41 \sim 0.45)$ & 0.09 & 0.926 \\
\hline KPS & $-1.51(-5.07-2.05)$ & -0.85 & 0.399 \\
\hline Nutritional status assessment & & & \\
\hline NRS2002 & $-0.38(-0.70 \sim-0.07)$ & -2.47 & $\mathbf{0 . 0 1 7}$ \\
\hline PG-SGA & $-1.56(-2.67$ to -0.44$)$ & -2.8 & $\mathbf{0 . 0 0 7}$ \\
\hline Appetite assessment & & & \\
\hline FAACT-A/CS & $2.31(0.50 \sim 4.12)$ & 2.56 & $\mathbf{0 . 0 1 4}$ \\
\hline Brief Dietary Self-Assessment Scale & $-0.04(-0.37 \sim 0.29)$ & -0.25 & 0.806 \\
\hline Psychological assessment & & & \\
\hline DT & $-1.08(-1.68$ to -0.47$)$ & -3.57 & $<0.001$ \\
\hline QoL assessment & & & \\
\hline EQ-5D index score & $0.04(0.00-0.07)$ & 2.14 & $\mathbf{0 . 0 3 7}$ \\
\hline EQ-VAS & $6.71(2.31-11.11)$ & 3.06 & $\mathbf{0 . 0 0 4}$ \\
\hline$p$ values < 0.05 are presented in bold face & & \\
\hline
\end{tabular}

\subsection{Change Trend of Various Indicators According to the Number of Visits}

The GLM was used to analyze the change trend of various indicators according to the number of visits (Fig. 2 and Fig. 3). The PG-SGA and DT scores significantly decreased with increasing number of visits ( $p$ 
$<0.05)$, suggesting that nutritional and psychological status gradually improved with increasing duration of symptom management. The EQ-VAS can better reflect the changes in QoL and showed a significant change with increasing numbers of visits $(p=0.002)$, indicating that patient health status improved with symptom management. Other indicators failed to display significant differences $(p>0.05)$, which may be related to the small sample size and short observation time during the analysis.

\section{Discussion}

\subsection{An Effective Symptom Management Model Has Been Established Based On ePROs}

In this project, a closed-loop symptom management model was constructed based on ePROs and was used to monitor the symptoms and QoL of patients with advanced gastrointestinal cancer. The preliminarily findings indicate that after nearly 3 months of symptom management, the model can significantly improve nutritional status and appetite, relieve depression and anxiety, maintain weight and $\mathrm{BMI}$, and maintain the physical status of the patients. Additionally, improvement in QoL was observed. This result suggested that the application of this model could optimize the effect of anticancer treatment and increase clinical benefits. These benefits may be due to the fact that ePROs can greatly promote the amount of attention patients give to their symptoms, enhance doctor-patient communication, and enable some patients to adjust and improve in a timely manner under the guidance of doctors.

\section{2 ePRO-based Symptom Management Model Can Complement Missing Information for Clinical Treatments and Promote Patient Education}

At present, symptom judgment is mainly completed by doctors. It is easy to ignore some symptoms, and there is not a good evaluation criteria for the severity of symptoms. Basch et al ${ }^{[23]}$ conducted a study in which clinicians and patients reported the occurrence of six symptoms, and found that there were large differences in the reported results, and clinicians often underestimated the occurrence of symptoms. PROs can provide more relevant information on the incidence and severity of symptoms. Therefore, some studies suggest using PROs to collect symptom information systematically as a way to improve symptom control ${ }^{[24-25]}$.

In this study, a model based on ePROs was shown to effectively detect and evaluate patients' symptoms, provide doctors with multidimensional information of patients in a convenient way, and supplement missing information related to nutrition, appetite, psychology and QoL. This missing information can help to understand the patients' more comprehensive condition and formulate individualized treatment plans, Additionally, patient education content can be shared regularly based on assessment results. This model helps to increase the awareness and attention of physicians and patients about related symptoms, complements the missing information required for clinical treatment and promotes patient education, which will be more widely implemented subsequently. 


\section{3 ePRO-based Symptom Management Model Prompts More Attention to the Nutritional and Dietary Problems of Patients}

Patients with malignant tumors often experience varying degrees of malnutrition, and negative energy balance and skeletal muscle loss can be observed in these patients, driven by a combination of reduced food intake and metabolic disturbances. Therefore, malnutrition in cancer patients cannot be cured by short-term nutritional correction. The European Society for Clinical Nutrition and Metabolism (ESPEN) nutritional guidelines recommend that all cancer patients should undergo regular nutritional assessment to determine the need for nutritional intervention and detect the impact of nutrition [26].

Anorexia is also common in cancer patients. Approximately $50 \%$ of newly diagnosed patients have anorexia, and in patients with advanced cancer, the rate is as high as $80 \%$ [27]. Anorexia will further aggravate malnutrition and lead to cachexia. In fact, in cancer patients, anorexia, malnutrition and cachexia often coexist. Therefore, enough attention should be given to the management of appetite and dietary guidance.

In addition, dietary problems are common in cancer patients. The results of the first visit in this study showed that the patients' dietary status was poor. After 3 treatment cycles of symptom management, most indicators were significantly improved, but the improvement of dietary problems was limited. This finding indicates that further attention should be given to dietary issues. Correct dietary guidance and education should be strengthened to ensure that patients can ingest adequate and structurally reasonable nutrition.

\subsection{Strengths}

Our study has several strengths. First, based on PRO, a multidisciplinary symptom management closedloop model was established. This model can help clinicians detect patients' symptoms early, improve symptom control, improve QoL, and help clinicians better manage patients. Second, the model we constructed is relatively simple and easy to implement and can be combined with the existing work of medical staff, so that it can be easily extended to daily diagnosis and treatment. Finally, an ePRO system was established. Scale completion, scoring and data management were completed through the system, which improved the convenience of the use of this model, saved management costs, and conformed to the current trend of medical digitization and intelligence.

\subsection{Limitations}

The limitations of this project are as follows. First, the closed-loop part of the symptom management model needs to be further improved and optimized. Therefore, we plan to strengthen the ePRO system construction and subsequently improve functions. For example, when the patients' results are abnormal, the ePRO system will send a warning message to clinicians. In addition, symptom intervention currently mainly relies on clinicians. In the future, we plan to develop personalized guidance based on medical 
evidence, and then provide them to relevant patients through the system to assist doctors in symptom management. Through the above measures, we hope to further improve the closed-loop part and bring more therapeutic benefits to patients. Second, this project was conducted in a single institution that included patients mostly from Shaanxi Province. The education level, comprehension ability, compliance, economic status, and the representativeness of diet and lifestyle habits of the included patients and their families have certain limitations. With the improvement and expansion of the system and relying on the promotion of the Northwest Society for Clinical Research of Gastrointestinal Cancer, we plan to include more centers in studies in the future, which will help us to better understand in-depth topics and serve patients.

\section{Conclusion And Prospects \\ 5.1 Conclusion}

We use the REDCap platform to build the ePRO system, which is convenient to use and has diversified functions. Based on this system, a closed-loop model of symptom management has been established and its effectiveness has been preliminarily confirmed, it can slow down the exacerbation of symptoms, maintain or even improve the symptoms of patients as well as QoL, and result in the maintenance of performance status, thereby improving patient tolerability of antineoplastic therapies. The application of this model in daily diagnosis and treatment can help clinicians better monitor patient symptoms and manage patients.

\subsection{Prospects}

The key work in the next stage should include (1) improving the ePRO system to allow it to have more functions, such as sending warning information and pushing personalized guidance recommendations to optimize the closed-loop process; (2) expanding the application range of the ePRO system and symptommanagement model, combining multiple types of clinical studies to observe health-related contents and increase the breadth and depth of studies; and (3) expanding the scope of studies, relying on the

Northwest Society for Clinical Research of Gastrointestinal Cancer, including more sites and increasing the study population, with the aim of bringing clinical benefits to more patients.

\section{Declarations}

Funding No funding was received for conducting this study.

Conflicts of interest/Competing interests The authors declare that they have no conflicts of interest

Availability of data and material The authors have full control of all primary data and agree to allow the journal to review the data if requested

Code availability Not applicable 
Authors' contributions All authors contributed to the study conception and design. Xuyuan Dong, Huili Wu, Yangwei Fan, Miaomiao Zhang and Xiaofang Chen were involved in the collection of data. Xuyuan Dong, and Yinying Wu contributed to the interpretation of the data and the preparation and writing of the manuscript. All authors approved the final manuscript.

Ethics approval The trial was approved by the Ethics Committee of the First Affiliated Hospital of Xi'an Jiaotong University (reference number XJTU1 AF2021LSK-197) and was performed in line with the principles of the Declaration of Helsinki

Consent to participate Verbal informed consent was obtained prior to the study

Consent for publication Not applicable

\section{References}

1. Sung H, Ferlay J, Siegel RL et al (2021) Global cancer statistics 2020: GLOBOCAN estimates of incidence and mortality worldwide for 36 cancers in 185 countries. CA Cancer J Clin 71(3):209-249. https://doi.org/10.3322/caac.21660

2. Martin L, Senesse P, Gioulbasanis I, Antoun S, Bozzetti F, Deans C, Strasser F, Thoresen L et al (2015) Diagnostic criteria for the classification of cancer-associated weight loss. J Clin Oncol 33(1):90. https://doi.org/10.1200/JC0.2014.56.1894

3. Abraham M , Kordatou Z, Barriuso J et al(2019) Early recognition of anorexia through patientgenerated assessment predicts survival in patients with oesophagogastric cancer. PLoS ONE 14(11):e0224540. https://doi.org/10.1371/journal.pone.0224540

4. Basch E (2017). Patient-Reported Outcomes - Harnessing Patients' Voices to Improve Clinical Care. N Engl J Med 376(2):105-108. https://doi.org/10.1056/NEJMp1611252.

5. U.S. Department of Health and Human Services FDA Center for Drug Evaluation and Research., U.S. Department of Health and Human Services FDA Center for Biologics Evaluation and Research. \& U.S. Department of Health and Human Services FDA Center for Devices and Radiological Health (2006) Guidance for industry: patient-reported outcome measures: use in medical product development to support labeling claims: draft guidance. Health and Quality of Life Outcomes 4(1):79. https://doi.org/10.1186/1477-7525-4-79

6. Calvert M, Kyte D, Mercieca-Bebber R et al (2018) Guidelines for Inclusion of Patient-Reported Outcomes in Clinical Trial Protocols: The SPIRIT-PRO Extension. JAMA 19(5):483-494. https://doi.org/10.1001/jama.2017.21903

7. Cleeland CS, Wang XS, Shi Q, et al (2011) Automated symptom alerts reduce postoperative symptom severity after cancer surgery: a randomized controlled clinical trial. J Clin Oncol, 29(8): 994-1000. https://doi.org/10.1200/JC0.2010.29.8315

8. Basch E, Deal AM, Kris MG et al (2016) Symptom Monitoring with Patient-Reported Outcomes During Routine Cancer Treatment: A Randomized Controlled Trial. J Clin Oncol 34(6):557-565. 
https://doi.org/10.1200/JC0.2015.63.0830

9. Basch E, Artz D, Dulko D et al (2005) Patient online self-reporting of toxicity symptoms during chemotherapy. J Clin Oncol 23(15):3552. https://doi.org/10.1200/JC0.2005.04.275

10. Jensen RE, Rothrock NE, Dewitt EM et al (2015) The role of technical advances in the adoption and integration of patient-reported outcomes in clinical care. Med Care 53(2):153-159.

https://doi.org/10.1097/MLR.0000000000000289

11. Howell D, Molloy S, Wilkinson K et al (2015) Patient-reported outcomes in routine cancer clinical practice: a scoping review of use, impact on health outcomes, and implementation factors. Ann Oncol 26(9):1846-1858. https://doi.org/10.1093/annonc/mdv181

12. Lu Z, Fang Y, Liu C et al (2021) Early Interdisciplinary Supportive Care in Patients With Previously Untreated Metastatic Esophagogastric Cancer: A Phase III Randomized Controlled Trial. J Clin Oncol 39(7):748-756. https://doi.org/10.1200/JC0.20.01254

13. Qiong Zhang, Yize Li, Lei Wen, Hongmei Zhang (2019) The Value and Significance of Patient Reported Outcome in the Treatment of Colorectal Cancer. Advances in Clinical Medicine 9(4):464469. https://doi.org/10.12677/acm.2019.94072

14. Kondrnp J, Rasmussen HH, Hamberg $\mathrm{O}$ et al (2003) Nutritional risk screening (NRS 2002): a new method based on an analysis of controlled Clinical trials. Clin Nutr 22 (3):321-336. https://doi.org/10.1016/s0261-5614(02)00214-5

15. Bauer J, Capra S, Ferguson ML (2002) Use of the scored Patient-Generated Subjective Global Assessment (PG-SGA) as a nutrition assessment tool in patients with cancer. European Journal of Clinical Nutrition 56(8):779-785. https://doi.org/10.1038/sj.ejcn.1601412

16. Adriana ADT, Misino P, Giannantoni P et al (2013) Comparison of the performance of four different tools in diagnosing disease-associated anorexia and their relationship with nutritional, functional and clinical outcome measures in hospitalized patients. Clinical Nutrition 32(4):527-532. https://doi.org/10.1016/j.clnu.2012.11.011

17. Minghua Cong, Hanping Shi (2018) The invention of a simple diet selfassessment tool for cancer patients. Electron J Metab Nutr Cancer 5(1):11-13. https://doi.org/10.16689/j.cnki.cn119349/r.2018.01.002

18. S. Blauwhoff-Buskermolen, C. Ruijgrok, R. W. Ostelo et al (2016) The assessment of anorexia in patients with cancer: cut-off values for the FAACT-A/CS and the VAS for appetite. Support Care Cancer 24:661-666. https://doi.org/10.1007/s00520-015-2826-2

19. Yening Zhang, Haiwei Zhang, Lili Song et al (2010) Application of the NCCN Distress Thermometer in Chinese cancer patient. Chinese Mental Health Journal 24(012):897-902. https://doi.org/10.1007/s11670-011-0054-y

20. Miller, Richard M (1990) EuroQol--a new facility for the measurement of health-related quality of life. Health Policy 16(3):199-208. https://doi.org/10.1016/0168-8510(90)90421-9

21. Dong Chen, Xiaoyu Li, Jing Luan (2019) Health Inequality and Its Influencing Factors among Middleaged and Elderly People in Urban and Rural Areas: Evidence from Health Survey Data Based on EQ- 
5D Scale. Statistics \& Information Forum 34(04):42-51. https://doi.org/10.3969/j.issn.10073116.2019.04.006

22. Hui Geng, Hairong He, Xiaotao Zeng, Jun Lv (2017) Application and Erection of REDCap System for Multi-center Clinical Data Acquisition System. Chin J Evid Based Cardiovasc Med 9(9):1025-1028. https://doi.org/10.3969/j.issn.1674-4055.2017.09.01

23. Basch E (2010) The missing voice of patients in drug-safety reporting. N Engl J Med 362(10):865. https://doi.org/10.1056/NEJMp0911494

24. Snyder CF, Aaronson NK, Choucair AK et al (2012) Implementing patient-reported outcomes assessment in clinical practice: A review of the options and considerations. Qual Life Res 21(8):1305-1314. https://doi.org/10.2307/41684666

25. Fung $\mathrm{CH}$, Hays RD (2008) Prospects and challenges in using patient-reported outcomes in clinical practice. Qual Life Res 17(10):1297-1302. https://doi.org/10.1007/s11136-008-9379-5

26. Laviano A, Meguid MM, Rossi-Fanelli F (2003) Cancer anorexia: clinical implications, pathogenesis, and therapeutic strategies. Lancet Oncology 4(11):686-694. https://doi.org/10.1016/S14702045(03)01247-6

27. Jann A, Patrick B, Vickie B et al (2017) ESPEN guidelines on nutrition in cancer patients. Clinical Nutrition 36(1):11-48. https://doi.org/10.1016/j.clnu.2016.07.015

\section{Figures}

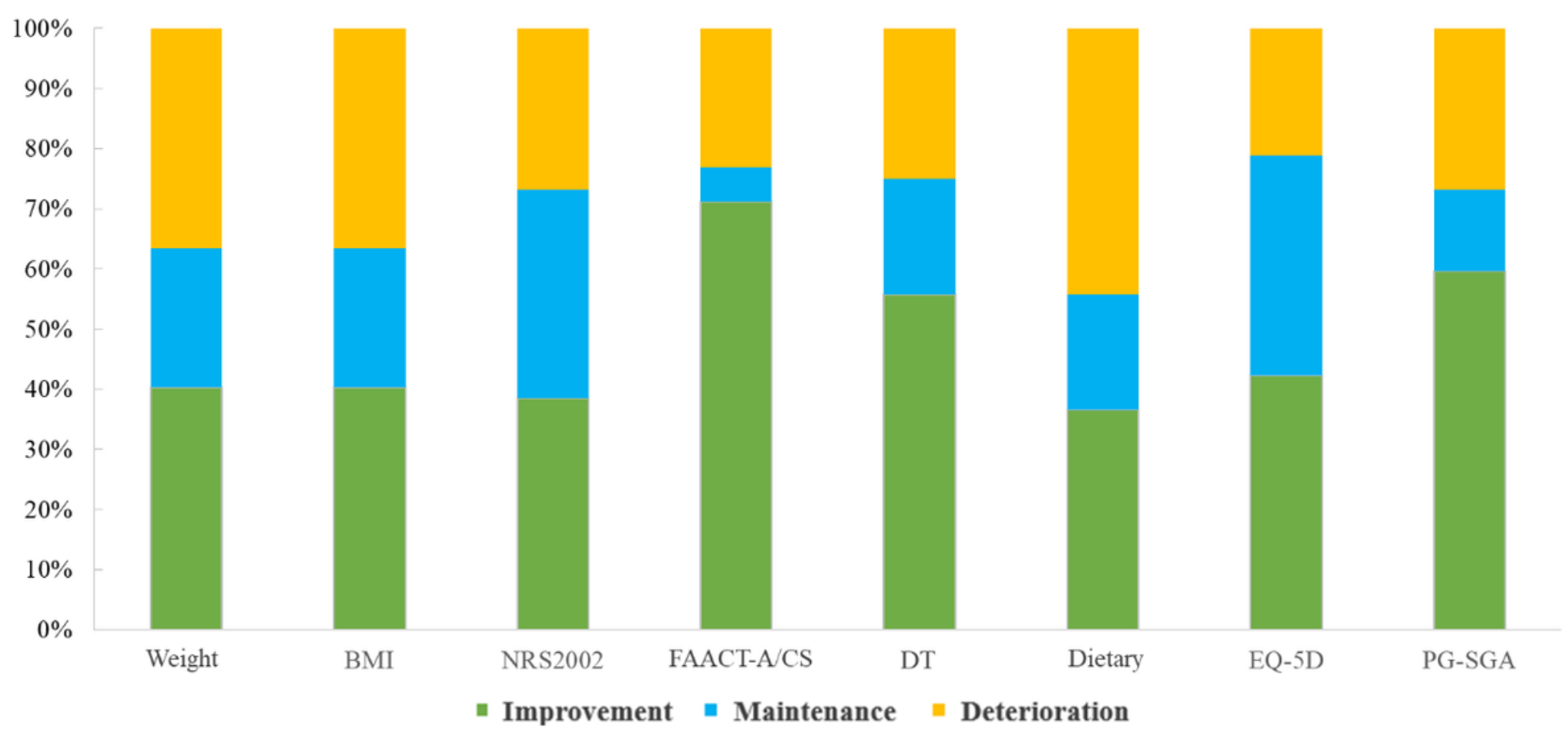

\section{Figure 1}

Proportion of patients with changes at Visit 4 compared with Visit 1 


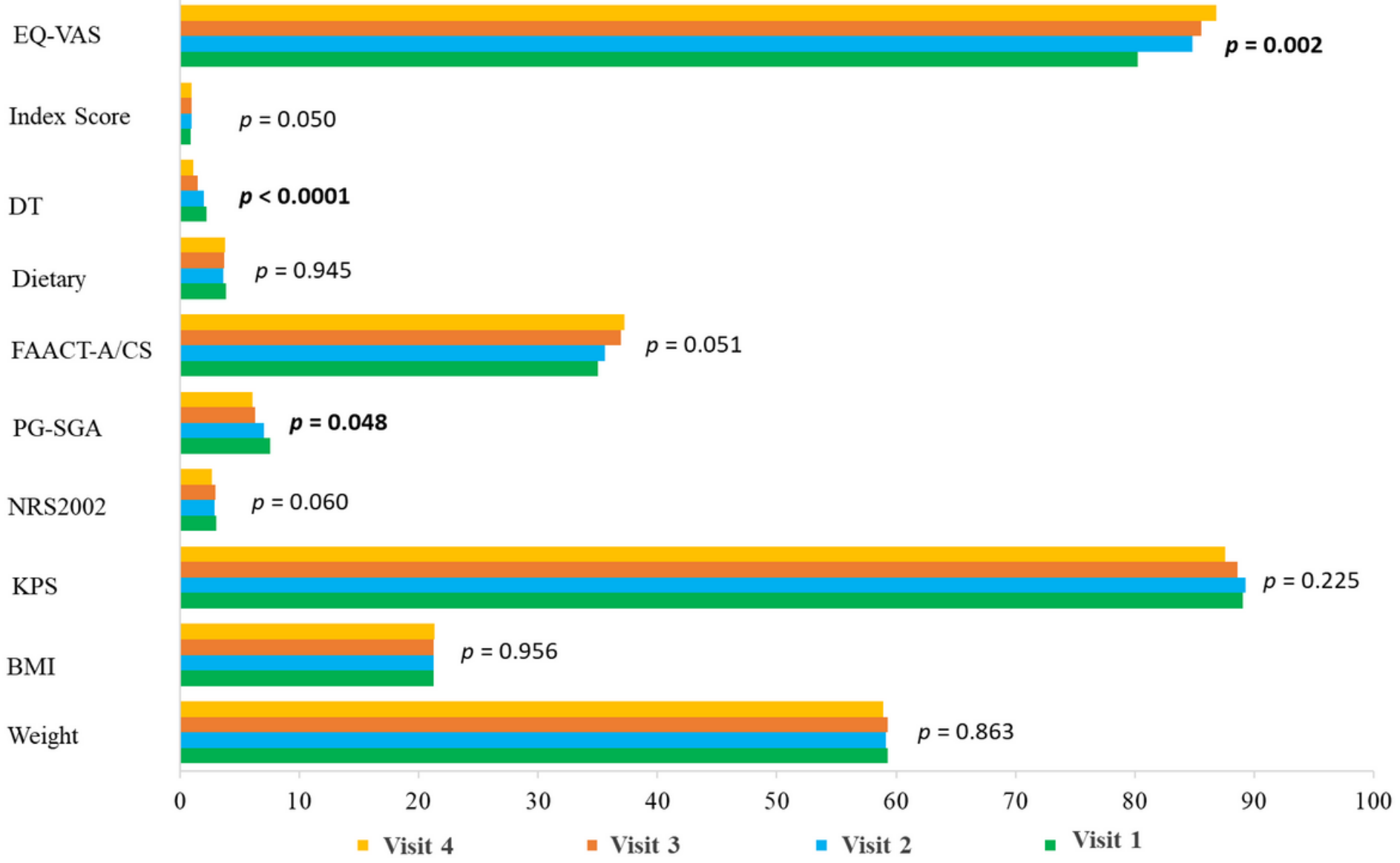

\section{Figure 2}

Bar chart of the change trend of various indicators according to the number of visits $p$ values $<0.05$ are presented in bold face

\section{Supplementary Files}

This is a list of supplementary files associated with this preprint. Click to download.

- APPENDIX1.docx 